\title{
ESTRUTURA DIAMÉTRICA DE UM FRAGMENTO DE FLORESTA OMBRÓFILA MISTA, EM ESTÁGIO INICIAL DE REGENERAÇÃO SOB DOMÍNIO DA ESPÉCIE Ligustrum lucidum W.T. Aiton
}

\author{
DIAMETER STRUCTURE OF A TROPICAL MIXED FOREST FRAGMENT IN \\ MIDDLE STAGE OF REGENERATION COLONIZED BY THE INVASIVE SPECIES \\ Ligustrum lucidum W.T. Aiton
}

\author{
Jonathan William Trautenmüller ${ }^{1}$, Juliane Borella², Antônio Pedro Fragoso Woycikievicz ${ }^{3}$, \\ Sérgio Costa Junior ${ }^{4}$, Myrcia Minatti ${ }^{5}$ \\ 1, 2, 3, 4, 5 Universidade Federal do Paraná, Curitiba, Paraná, Brasil-jwtraute@gmail.com, \\ borella.juli@gmail.com, antoniof.w@gmail.com, o.sergio.costa@gmail.com \& \\ myrciaminatti@gmail.com
}

\section{RESUMO}

\begin{abstract}
A Mata Atlântica é um bioma composto por inúmeras tipologias florestais distintas, dentre elas a Floresta Ombrófila Mista (FOM). A FOM apresenta-se de maneira reduzida e fragmentada em diferente estágio de regeneração, o que favorece a presença de espécies invasoras, como por exemplo, o Ligustrum lucidum W. T. Aiton. A hipótese proposta foi que a floresta e a espécie Ligustrum lucidum W. T. Aiton apresentam distribuição diamétrica decrescente ou unimodal. Dessa forma, o objetivo foi verificar a estrutura diamétrica da floresta e de Ligustrum lucidum. Para o estudo foram instaladas três unidades amostrais retangulares de $300 \mathrm{~m}^{2}$ em que todos os indivíduos com diâmetro à altura do peito (DAP) acima de $10 \mathrm{~cm}$ foram mensurados. Tanto para a floresta como para a espécie em questão, foram ajustadas funções probabilísticas (fdps), com o número de classes definidos pela metodologia de Sturges para avaliar seu comportamento, tendo seus ajustes avaliados através do teste de Kolmogorov-Smirnov. Os resultados mostraram que todas as funções apresentaram aderência ( $\alpha=5 \%)$ para os dados reais, tanto, para a floresta como para a espécie Ligustrum lucidum. Porém, quando considerada a ponderação dos ajustes para a floresta e para a espécie Ligustrum lucidum a função Wakeby apresentou a melhor bondade de ajuste no ranqueamento total. A estrutura diamétrica demonstra o estágio inicial de regeneração da floresta. A floresta e a espécie Ligustrum lucidum apresentam distribuição exponencial negativa em forma de "J" invertido, a curva do Ligustrum lucidum é mais achatada que a curva da floresta.
\end{abstract}

PALAVRAS-CHAVE: Funções probabilísticas, Mata atlântica, Modelagem.

\section{ABSTRACT}

The Atlantic Forest biome consists of a number of distinct forest typologies, including the Tropical Mixed Forest (TMF). It is commonly found in small areas and different stages of regeneration, which favors the presence of invasive species, such as Ligustrum lucidum W. T. Aiton. The hypothesis proposed in this study was that the diameter distribution may be decreasing or unimodal for the forest and the species under study. Therefore, this work aimed to evaluate the diameter structure of both the forest community and the species Ligustrum lucidum. For the study were installed three rectangular sample units of $300 \mathrm{~m}^{2}$ in which all individuals with a diameter at breast height (DBH) above $10 \mathrm{~cm}$ were measured. For both the forest and the species in question, probabilistic functions (fdps) were adjusted, with the number of classes defined by Sturges methodology to evaluate their behavior, and their adjustments were evaluated through the Kolmogorov-Smirnov test. The results indicate that all the functions showed adherence $(\alpha=5 \%)$ for real data, both for the forest and for the species Ligustrum lucidum. However, when considering the adjustment weighting of the forest and the species Ligustrum lucidum, the Wakeby function showed the best goodness of fit in the total ranking. The diameter structure demonstrates the early stage of regeneration of the forest fragment under study. The forest and the species Ligustrum lucidum have negative exponential distribution in an inverted "J" shape. The curve of the species Ligustrum lucidum is flatter than the curve of the forest. 


\section{INTRODUÇÃO}

O Bioma Mata Atlântica, devido a expansão das fronteiras agrícolas e crescimento populacional, teve uma redução da cobertura original, de $15 \%$ para $7,7 \%$ do território nacional (OLIVEIRA, 2007). Na região sul e sudeste do Brasil essa distribuição da Mata Atlântica encontra-se distribuída em diversos fragmentos menores, ou seja, há poucas áreas de grande extensão, e as mesmas em diversos estágios de regeneração (ZAÚ, 1998). A Floresta Ombrófila Mista - FOM, é considerada uma das mais importantes tipologias florestais da Mata Atlântica (RODE et al., 2009). A FOM possui espécies de grande importância ecológica e/ou econômica, como Araucaria angustifolia (Bertol.) Kuntze, Ocotea porosa (Nees) Barroso, Ilex paraguariensis A. St.-Hil, Eugenia uniflora L.

Contudo, boa parte dos fragmentos da FOM encontram-se em diferentes estádios de regeneração, devido principalmente a perturbações de natureza antrópicas (KLAUBERG et al., 2010). Nesse sentido, muitas espécies nativas encontram barreiras para sua regeneração natural, como a competição por espaço, luz, água e nutrientes com espécies exóticas invasoras (RODE et al., 2009). Dessa forma, o estabelecimento de espécies exóticas invasoras nos ambientes naturais em regeneração é uma das causas da degradação da biodiversidade (NUNES et al., 2018).

Em fragmentos da FOM que se encontram em estádio inicial e/ou médio de regeneração tem sido constatada a presença de espécies exóticas invasoras, entre elas o Ligustrum lucidum W.T. Aiton (GUIDINI et al., 2014; RODRIGUES et al., 2015; MALIZIA et al., 2017). Essa espécie foi introduzida no país para fins ornamentais, principalmente em ambientes urbanos (GRAMIGNOLLI et al., 2016).

O Ligustrum lucidum é conhecida popularmente como ligustro ou alfeneiro, trata-se de uma planta de origem asiática, mais especificamente do sudoeste da China, a qual possui uma boa dispersão ocasionada por pássaros atraídos pelos frutos da espécie (MATTHEWS, 2005; BIONDI \& PEDROSA-MACEDO, 2008). De acordo com Theoharides \& Dukes (2007) e Roques (2012), a invasão de espécies exóticas pode acarretar em alterações na vegetação dominante, uma vez que, geralmente as espécies exóticas invasoras são mais rústicas quando comparadas as nativas.

Para avaliar esse potencial invasor, os estudos sobre a estrutura diamétrica tem se tornado uma das ferramentas para descrever a uniformidade e o crescimento de uma floresta em comparação com as espécies invasoras. Esses estudos fornecem informações indispensáveis para inventários florestais em diferentes níveis de estrutura e dinâmica da área em relação à variabilidade da densidade dentro das classes de tamanho (ASSMANN, 1970). Se a distribuição do diâmetro representar um único pico com distorção da concentração de densidade para a esquerda, este padrão deve ser mantido apesar das intervenções silvícolas (ZHENG \& ZHOU, 2010).

Nesse sentindo, modelos matemáticos têm sido utilizados para controle, projeção e conhecimento da estrutura da população florestal, tendo as funções probabilísticas como uma das principais ferramentas na modelagem estrutural de florestas homogêneas e heterogêneas (NASCIMENTO et al., 2012). Arce (2004) ressalta que essa é uma das ferramentas de mais fácil aplicação para caracterizar a estrutura de um fragmento ou povoamento florestal.

Dessa forma, este trabalho teve como objetivo avaliar a estrutura diamétrica de um fragmento de FOM em estágio inicial de regeneração com o uso de funções probabilísticas e da população presente da espécie Ligustrum lucidum.

\section{MATERIAL E MÉTODOS}

A área de estudo está localizada no município de Rio Branco do Sul, Paraná, Brasil, com três hectares. Essa área foi utilizada para produção agrícola, porém foi abandonada em meados da década de 1990, a vegetação original é a Floresta Ombrófila Mista. A classificação climática segundo Köppen, é subtropical úmido ( $\mathrm{Cfb}$ ) com média anual entre 1.600 e $1.900 \mathrm{~mm}$ de chuva e temperatura média entre 16 e $18^{\circ} \mathrm{C}$ (ALVARES et al., 2013).

Os dados para a realização desse trabalho foram obtidos por meio de inventário florestal, em que, foram instaladas três parcelas retangulares de 10 m x 30 m (300 $\mathrm{m}^{2}$ ) distribuídas de forma aleatória no fragmento. Todas as árvores com diâmetro à altura do peito (DAP) maior que 10 $\mathrm{cm}$ foram mensuradas. Para a modelagem da estrutura do diâmetro, foram ajustadas funções probabilísticas (fdps) apresentadas na Tabela 1. As seguintes estatísticas descritivas foram determinadas: média, moda, mediana, variância, desvio padrão, amplitude, assimetria e curtose. Os parâmetros das Funções de Densidade de Probabilidade (fdps) foram obtidos por máxima verossimilhança através dos pacotes de MASS e fitdistrplus do software R. 
Tabela 1. Funções probabilísticas ajustadas para dados de distribuição diamétricas em área de regeneração natural sob domínio da Floresta Ombrófila Mista, localizado em Rio Branco do Sul - PR.

\begin{tabular}{|c|c|c|}
\hline Denominação & Funções probabilísticas & Condições \\
\hline Beta & $f(x)=\frac{1}{\beta\left(\alpha_{1}, \alpha_{2}\right)} \frac{(x-a)^{\alpha_{1}-1}(b-x)^{\alpha_{2}-1}}{(b-a)^{\alpha_{1}+\alpha_{2}-1}}$ & $\begin{array}{c}\alpha_{1}>0, \alpha_{2}<0, a<b \\
a \leq x \leq b\end{array}$ \\
\hline Exponencial 2P & $f(x)=\lambda e^{(-\lambda(x-\gamma))}$ & $\gamma \leq x<+\infty$ \\
\hline Gamma 3P & $f(x)=\frac{(x-\gamma)^{\alpha-1}}{\beta^{\alpha} \Gamma(\alpha)} e^{(-(x-\gamma) / \beta)}$ & $\begin{array}{c}\alpha>0, \beta>0 \\
\gamma \equiv 0\end{array}$ \\
\hline Log Normal 3P & $f(x)=\frac{e^{\left[-\frac{1}{2}\left(\frac{\ln (x-\gamma)-\mu}{\sigma}\right)^{2}\right]}}{(x-\gamma) \sigma \sqrt{2 \pi}}$ & $\begin{array}{c}\sigma>0 \\
\gamma \leq x<+\infty\end{array}$ \\
\hline SB Johnson & $f(x)=\frac{\delta}{\lambda \sqrt{2 \pi} z(1-z)} e^{\left(-\frac{1}{2}\left(\gamma+\delta \ln \left(\frac{z}{1-z}\right)\right)^{2}\right)}$ & $\begin{array}{l}\alpha>0 \\
\beta>0\end{array}$ \\
\hline Wakeby & $f(x)=\xi+\frac{\alpha}{\beta}\left(1-(1-F)^{\beta}\right)-\frac{\gamma}{\delta}\left(1-(1-F)^{-\delta}\right)$ & $\begin{array}{c}\alpha \neq 0 \text { ou } \gamma \neq 0, \beta+\delta>0 \text { ou } \beta=\gamma= \\
\delta=0, \text { se } \alpha=0 \text {, então } \beta=0 \\
\text { se } \gamma=0 \text {, então } \delta=0, \gamma \geq \alpha+\gamma \geq 0\end{array}$ \\
\hline Weibull 3P & $f(x)=\frac{\alpha}{\beta}\left(\frac{x-\gamma}{\beta}\right)^{\alpha-1} e^{\left[-\left(\frac{x-\gamma}{\beta}\right)^{\alpha}\right]}$ & $\gamma \leq x<+\infty$ \\
\hline
\end{tabular}

Em que: $\mathrm{k}, a, b, \alpha, b, \gamma, \mu, \lambda, \delta, \xi=$ parâmetros; $x=$ centro de classe diamétrica; $\pi, e=$ constante; $\mu$ = média aritmética; $\sigma=$ desvio padrão; In = logaritmo neperiano; e $\Gamma$ = função Gamma.

O número de classes de diâmetro foi definido pelo método de Sturges (STURGES, 1926), sendo:

$$
K=1+3,33(\log n)
$$

Em que: $K=$ Números de classes; e $n=$ Número de dados amostrais.

Os intervalos de classe foram definidos com base na amplitude diamétrica da floresta e do Ligustrum lucidum. A qualidade do ajuste foi avaliada por meio do teste de Kolmogorov-Smirnov (SCHNEIDER et al., 2009), ao nível de 95\% de probabilidade. Este teste foi utilizado para verificar as seguintes hipóteses do teste bilateral: $\mathrm{H}_{0}=$ os diâmetros observados seguem as distribuições propostas; e $\mathrm{H}_{1}=$ os diâmetros observados não seguem as distribuições propostas.

As melhores funções foram escolhidas por meio dos escores estatísticos com o objetivo de resumir os resultados e facilitar o processo de seleção. O valor ponderado foi determinado atribuindo pesos à estatística calculada, sendo o peso um para a função com melhor aderência e pesos crescentes para as fdps restantes.

\section{RESULTADOS E DISCUSSÃO}

A estatística descritiva (Tabela 2) evidenciou que para a floresta e para Ligustrum lucidum o diâmetro máximo não ultrapassa os $30 \mathrm{~cm}$, demonstrando que a floresta se encontra em estágio inicial de regeneração natural. A média $(16,48 \mathrm{~cm})$ e mediana $(15,99 \mathrm{~cm})$ dos diâmetros foi maior para o Ligustrum lucidum quando comparados com a floresta (média $=15,04 \mathrm{~cm}$, mediana $=13,69 \mathrm{~cm}$ ).

Tabela 2. Estatísticas descritivas do DAP para a floresta e Ligustrum lucidum em área de regeneração natural sob domínio da Floresta Ombrófila Mista em Rio Branco do Sul - PR.

\begin{tabular}{ccc}
\hline Medida & Floresta & Ligustrum lucidum \\
\hline N.ind $^{-1}$ & 143 & 68 \\
\hline Média $(\mathrm{cm})$ & 15,04 & 16,48 \\
\hline Mediana $(\mathrm{cm})$ & 13,69 & 15,99 \\
\hline Moda $(\mathrm{cm})$ & 10,19 & 10,19 \\
\hline Dmin $(\mathrm{cm})$ & 10,03 & 10,19 \\
\hline Dmax $(\mathrm{cm})$ & 29,28 & 29,28 \\
\hline Amplitude $(\mathrm{cm})$ & 19,25 & 10,09 \\
\hline Variância $\left(\mathrm{cm}{ }^{2}\right)$ & 18,31 & 23,03 \\
\hline Desvio Padrão $(\mathrm{cm})$ & 4,27 & 4,80 \\
\hline Assimetria & 1,18 & 0,75 \\
\hline Curtose & 1,09 & 0,06 \\
\hline
\end{tabular}

Em que: $\mathrm{N} \cdot$ ind $^{-1}=$ número de indivíduos avaliados; Dmin = diâmetro mínimo; e Dmax = diâmetro máximo.

A estatística descritiva mostra uma floresta bastante homogênea em estágio inicial de regeneração. Além disso, com a análise gráfica das distribuições diamétricas da floresta e da espécie Ligustrum lucidum (Figura 1) verificase que as duas seguem o padrão de florestas naturais com a forma exponencial negativa (" $\mathrm{J}$ " invertido). 

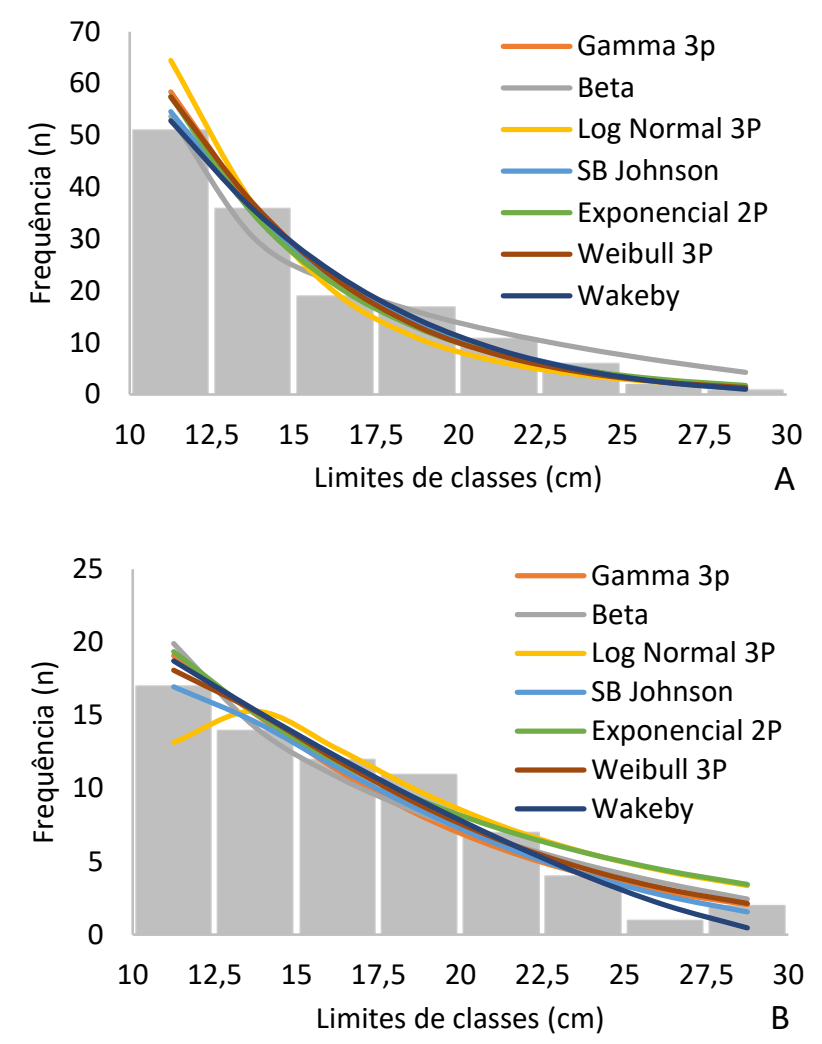

Figura 1. Distribuições diamétrica para a floresta $(A)$ e Ligustrum lucidum (B) em área de regeneração sob domínio da Floresta Ombrófila Mista em Rio Branco do Sul, PR.

Neste fragmento florestal o Ligustrum lucidum corresponde a $32 \%$ da densidade da floresta, quando comparado com os demais fragmentos de floresta ombrófila mista. Nos trabalhos realizados por Guidini et al. (2014) e Rodrigues et al. (2015) encontraram 13\% e 4\% da densidade, respectivamente.

Rodrigues et al. (2015) destaca o Ligustrum lucidum como espécie invasora de grande potencial, devido seu incremento em diâmetro e densidade, associado a baixa mortalidade indicam que a espécie apresenta boa adaptação frente as espécies nativas.

Na Tabela 3 estão apresentados todos os parâmetros das funções ajustadas para a floresta e a espécie Ligustrum lucidum. $\mathrm{O}$ teste de Kolmogorov-Smirnov e o ranqueamento da bondade de ajuste, referente as funções testadas para a floresta e a espécie Ligustrum lucidum estão representados na Tabela 4. É possível constatar que todas as funções apresentaram aderência ( $\alpha=5 \%$ ) para os dados reais, tanto, para a floresta como para a espécie Ligustrum lucidum.

A aderência das funções consegue representar a distribuição exponencial negativa. O que era esperado, devido a florestas naturais com regeneração em fluxo contínuo apresentam a forma de "J" invertido, como mencionado por vários pesquisadores (ORELLANA et al., 2017).

Para a floresta a função Wakeby apresentou melhor aderência seguida da função SB Johnson (Figura 1A). Outros trabalhos citam outras funções para floresta Ombrófila Mista como Weibull 3P (ORELLANA et al., 2017) e Weibull 2P (LIMA et al., 2017), no entanto, a função Wakeby não foi ajustada nesses estudos. Para a espécie Ligustrum lucidum a função Weibull $3 \mathrm{P}$ foi a que proporcionou melhor ajuste seguida da função Beta (Figura 1B). Porém, quando considerada a ponderação dos ajustes da floresta e da espécie Ligustrum lucidum a função Wakeby apresentou a melhor bondade de ajuste no ranqueamento total.

Para verificar as diferenças entre as distribuições da floresta e do Ligustrum lucidum foi selecionada a função com melhor bondade de ajuste pelo ranque total (Tabela 4). Na Figura 2 pode-se observar que acima do limite de classe de $22,5 \mathrm{~cm}$ o número de árvores estimado (ind.ha-1 ${ }^{-1}$ ) torna-se igual entre a floresta e o Ligustrum lucidum. Esse resultado é devido a presença única de Ligustrum lucidum nas classes de diâmetro acima de $22,5 \mathrm{~cm}$ de DAP.

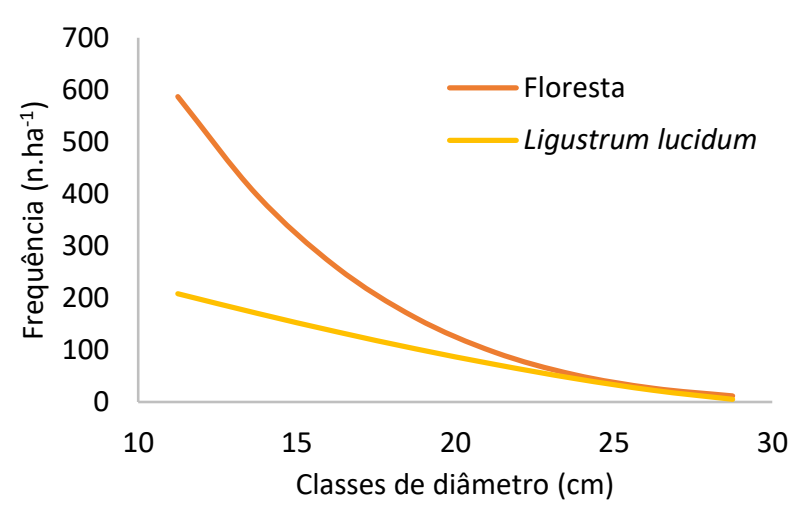

Figura 2. Distribuição diamétrica para a floresta e para o Ligustrum lucidum utilizando a função de Wakeby em área de regeneração natural sob domínio da Floresta Ombrófila Mista em Rio Branco do Sul, PR.

Quanto a bondade de ajuste, para a floresta e o Ligustrum lucidum, a função Wakeby apresentou a melhor aderência. Sanquetta et al. (2014) descreveram que a função Wakeby apresenta boa flexibilidade para se aderir a dados que apresentam grande frequência em apenas um centro de classe. A função Wakeby vem sendo utilizada para modelar eventos com frequências extremas em poucos centros de classes, como dados climáticos e hidrológicos (SANQUETTA et al., 2014), para os quais, as funções contínuas tradicionais apresentam grande dificuldade de se aderirem. 
Tabela 3. Parâmetros estimados das funções probabilísticas para a floresta e o Ligustrum lucidum em área de regeneração natural sob domínio da Floresta Ombrófila Mista em Rio Branco do Sul, PR.

\begin{tabular}{|c|c|c|c|c|c|c|c|c|c|}
\hline \multirow{2}{*}{ Funções } & \multicolumn{9}{|c|}{ Parâmetros } \\
\hline & $\alpha$ & $\beta$ & $\gamma$ & $\mu$ & $\lambda$ & $\delta$ & $\xi$ & $a$ & $b$ \\
\hline \multicolumn{10}{|c|}{ Floresta } \\
\hline Beta & 0,55491 & 2,0436 & - & - & - & - & - & 9,83 & 35,136 \\
\hline Exponencial 2P & - & - & 8,93 & - & 0,19946 & - & - & - & - \\
\hline Gamma 3P & 1,1135 & 4,5078 & 8,824 & - & - & - & - & - & - \\
\hline LogNormal 3P & 0,773 & - & 7,7726 & 1,4961 & - & - & - & - & - \\
\hline SB Johnson & - & - & 1,4555 & - & 26,26 & 0,92547 & 7,8677 & - & - \\
\hline Wakeby & 5,9548 & 0,18246 & 0 & - & - & 0 & 8,63 & - & - \\
\hline Weibull 3P & 1,103 & 5,1961 & 8,724 & - & - & - & - & - & - \\
\hline \multicolumn{10}{|c|}{ Ligustrum lucidum } \\
\hline Beta & 0,75417 & 2,6744 & - & - & - & - & - & 9,09 & 40,136 \\
\hline Exponencial 2P & - & - & 10,19 & - & 0,0988 & - & - & - & - \\
\hline Gamma 3P & 1,2599 & 6,4335 & 8,13 & - & - & - & - & - & - \\
\hline LogNormal 3P & 0,8649 & - & 8,5543 & 2,2393 & - & - & - & - & - \\
\hline SB Johnson & - & - & 1,2846 & - & 35,84 & 1,1013 & 5,0837 & - & - \\
\hline Wakeby & 3298,8 & 417,44 & 9,2393 & - & - & $-0,41637$ & 0,0 & - & - \\
\hline Weibull 3P & 1,2204 & 8,7942 & 8,086 & - & - & - & - & - & - \\
\hline
\end{tabular}

Em que: $a, b, \alpha, \beta, \gamma, \mu, \lambda, \delta$ e $\xi=$ parâmetros dos modelos.

Tabela 4. Teste de Kolmogorov-Smirnov sobre as funções testadas para a distribuição diamétrica para a floresta e Ligustrum lucidum em área de regeneração natural sob domínio da Floresta Ombrófila Mista em Rio Branco do Sul - PR.

\begin{tabular}{cccccc}
\hline \multirow{2}{*}{ Funções } & \multicolumn{2}{c}{ KS calculado } & \multicolumn{2}{c}{ Ranque } & \multirow{2}{*}{ Ranque Total } \\
\cline { 2 - 5 } & Floresta & Ligustrum lucidum & Floresta & Ligustrum lucidum & 6 \\
\hline Beta & $0,071^{* *}$ & $0,045^{* *}$ & 4 & 2 & 7 \\
Exponencial 2P & $0,053^{* *}$ & $0,121^{* *}$ & 3 & 3 & 8 \\
Gamma 3P & $0,074^{* *}$ & $0,046^{* *}$ & 5 & 6 & 13 \\
\hline LogNormal 3P & $0,105^{* *}$ & $0,056^{* *}$ & 7 & 5 & 7 \\
SB Johnson & $0,043^{* *}$ & $0,051^{* *}$ & 2 & 4 & 5 \\
Wakeby & $0,041^{* *}$ & $0,047^{* *}$ & 1 & 6 & 7 \\
Weibull 3P & $0,075^{* *}$ & $0,035^{* *}$ & 6 & 1 & 7 \\
\hline
\end{tabular}

Em que: KS = valores do teste de Kolmogorov-Smirnov; $\mathrm{e}^{* *}$ = significativo a $5 \%$ de probabilidade de erro.

A maior concentração de indivíduos para ambos, floresta e a espécie, nas classes iniciais aponta para um ciclo de vida curto com limite de $29 \mathrm{~cm}$ de DAP. Recorrente das características da área de estudo e do estágio de regeneração, indicando que a floresta está no início do processo de regeneração (AIDE et al., 2012; GUNKEL et al., 2013).

A generalização da função prova a necessidade do desenvolvimento de modelos individuais que melhor descrevem os dados. Assim, permitindo compreender as dinâmicas, os índices de competição, assimetria e a curtose da distribuição de áreas em estágio iniciais de regeneração.

\section{CONCLUSÕES}

A floresta e a espécie Ligustrum lucidum apresentam distribuição exponencial negativa em forma de "J" invertido.

A estrutura diamétrica demonstra o estágio inicial de regeneração do fragmento florestal.

Para a floresta e a espécie Ligustrum lucidum todas as funções estudadas foram aderentes.

\section{REFERÊNCIAS}

ALVARES, C.A. et al. Köppen's climate classification map for Brazil. Meteorologische Zeitschrift, v.22, n.6, p.711-728, 2013. 
AIDE, T.M. et al. Deforestation and reforestation of Latin America and the Caribbean (2001-2010). Biotropica, v.45, p.262-271, 2012.

ARCE, J.E. Modelagem da estrutura de florestas clonais de Populus deltoides March através de distribuições diamétricas probabilísticas. Ciência Florestal, v.14, p.149-164, 2004.

ASSMANN E. The principles of forest yield study. Oxford: Pergamon Press, 1970.

BIONDI, D.; PEDROSA-MACEDO, J.H. Plantas invasoras encontradas na área urbana de Curitiba (PR). Floresta, v.38, p.130-144, 2008.

GRAMIGNOLLI, V. et al. Arborização viária no bairro jardim das flores, no município de dourado (SP). Revista Brasileira Multidisciplinar, v.38, p.77-91, 2016.

GUIDINI, A.L. et al. Invasão por espécies arbóreas exóticas em remanescentes florestais no planalto Sul Catarinense. Revista Árvore, v.19, n.1, p.469-478, 2014.

GUNKEL, G. et al. Sustainable management of water and land in semiarid areas. 1.ed. Recife: Editora Universitária UFPE, 2013.

KLAUBERG, C. et al. Florística e estrutura de um fragmento de Floresta Ombrófila Mista no Planalto Catarinense. Biotemas, v.23, p.37-45, 2010.

LIMA, R.B. et al. Diameter distribution in a Brazilian tropical dry forest domain: predictions for the stand and species. Anais da Academia Brasileira de Ciências, v.89, n.2, p.1189-1203, 2017.

MALIZA, A. et al. Invasion of Ligustrum lucidum (Oleaceae) in subtropical secondary forest of NW Argentina: declining growth rates of abundant native tree species. Journal of Vegetation Science, v.28, p.1240-1249, 2017.

MATTHEWS, S. América do Sul invadida: a crescente ameaça das espécies exóticas invasoras. Nairobi: Global Invasive Species Programme, 2005.

NASCIMENTO, R.G.M. et al. Modelo de projeção por classe diamétrica para florestas nativas: enfoque na função probabilística de Weibull. Pesquisa Florestal Brasileira, v.32, p.209-219, 2012.

NUNES, A.S. et al. Ligustrum lucidum como uma espécie invasora oportunista em uma Floresta com Araucária no sul do Brasil. Rodriguésia, v.69, n.2, p.351-362, 2018.

OLIVEIRA, D.R. Mata atlântica, paleoterritórios e história ambiental. Ambiente \& Sociedade, v.10, p.11-23, 2007.

ORELLANA, E. et al. Métodos de ajuste e procedimentos de seleção de funções probabilísticas para modelar a distribuição diamétrica em floresta nativa de Araucária. Ciência Florestal, v.27, n.3, p.969-980, 2017.

RODE, R. et al. Comparação Florística entre uma Floresta Ombrófila Mista e uma vegetação arbórea estabelecida sob um povoamento de Araucaria angustifolia de 60 anos. Cerne, v.15, p.101-115, 2009.
RODRIGUES, A.L. et al. Dinâmica e modelagem autologística da distribuição da espécie invasora Ligustrum lucidum W. T. Ainton em floresta nativa. Scientia Forestalis, v.43, p.665-674, 2015.

ROQUES, A. Invasive species. Integrative zoology, v.7, n.3, p.227227, 2012.

SANQUETTA, C.R. et al. Probabilistic distributions for Acacia mearnsii de Wild total height and the influence of environmental factors. Journal of Applied Mathematicsand Physics, v.2, p.1-10, 2014.

SCHNEIDER, P.R. et al. Análise de Regressão aplicada à Engenharia Florestal. 2.ed. Santa Maria: FACOS, 2009.

STURGES, $\mathrm{H}$. The choice of a class interval. Journal of the American Statistical Association, v.21, n.153, p.65-66, 1926.

THEOHARIDES, K.A.; DUKES, J.S. Plant invasion across space and time: factors affecting nonindigenous species success during four stages of invasion. New Phytologist, v.176, n.2, p.256-273, 2007.

ZAÚ, A.S. Fragmentação da Mata Atlântica: aspectos teóricos. FLORAM, v.5, p.160-170, 1998.

ZHENG, L.; ZHOU, X. Diameter distribution of trees in natural stands managed on polycyclic cutting system. Forestry Studies in China, v.12, p.21-25. 2010. 